\title{
A case report: Amelogenesis imperfecta
}

Rabia Zia, Samreen Aqeel

\section{SUMMARY}

The aim of this case report is to come up with patient friendly restorative treatment for a young female patient with severe AI using indirect composite veneers for anteriors and metal crowns on posteriors. Amelogenesis imperfecta is defined as genetic determined malformation with various manifestations particularly concerning functional disability and enamel discoloration.

After the performed dental procedure, patient improved a lot in terms of chewing efficacy, which in turn enhance her quality of life. Her confidence level was also boosted up with acceptable esthetics. The patient was better able to maintain her oral hygiene in terms of plaque removal and tooth brushing. Clinical Relevance: Patients with amelogenesis imperfect needs life time follow up with high level of maintenance provided with the prosthesis. This particular management provided the patient with least invasive procedure in terms of simplicity and cost effectiveness.

The current case report exhibits that restorative treatment enhances the quality of life of patients. Some other aspects needs to be addressed depending upon the severity and affordability of patients, such as surgical correction of the jaws, and orthodontic treatment.

KEYWORDS: Amelogenesis Imperfect, Clinical Management, Indirect Restorations, Follow-Up,

Restorative treatment

\section{INTRODUCTION}

Amelogenesis Imperfecta (AI) is a hereditary derived enamel development disorder of enamel formation formed by ameloblasts that effects quality and quantity of tooth enamel. This usually affects permanent and deciduous dentitions. ${ }^{1}$ The diagnoses of AI is confirmed through various tests including, clinical examination, radiographic analysis and gene analysis. On clinical examination AI picture is presented as reduced enamel thickness or as with mottled enamel appearance or with brownish to greyish enamel discoloration. On the radiograph no enamel structure is seen on teeth or much reduced enamel structure is present ${ }^{3}$. This mutation can even pass from parents to children. It can even affect subjects with no family history. Previous literature showed that not a single gene alteration is responsible for this condition rather than multiple genes are responsible for causing this state. A study carried out

\footnotetext{
Dr. Rabia Zia, $B D S$

PGR Operative Dentistry

Fatima Memorial Hospital LHR

Dr. Samreen Aqeel, $B D S$

PGR Operative Dentistry

Fatima Memorial Hospital LHR

Correspondence:

Rabia Zia

Email: rabiya719@gmail.com
}

on Turkish families subjects who had hypomaturation amelogenesis imperfecta showing Whole-exome sequence analyses identified disease that causes mutations in each proband, and these mutations cosegregated with the amelogenesis imperfecta phenotype in all recruited members of each family. Gene analysis showed AMELX missense mutation that is responsible for increased exonic definition of exon 4 and the MMP20 mutation declines exonic definition of exon one. Subsequently these gene alterations elicits an alteration of exon usage during RNA splicing, causing the enamel malformations. ${ }^{2}$ It is found that depending on the study population AI prevalence ranged from 1:700 to 1:14,000.

The extracted data from the previous researches shows, the condition has some genetic patterns, such as autosomal dominant, recessive and rarely as $\mathrm{X}$ chromosome-linked inheritance. On the other hand, many researches shows that AI occurs when there is mutation in the genes. ${ }^{3}$ It depends upon the gene involved and at what time the mutation occurs, various alterations have been noted in enamel from superficial discoloration to complete absence of enamel formation.

According to phenotypic alteration, amelogenesis imperfecta can be classified as type I hypoplastic, type II hypomatured, type III hypocalcified, and type IV hypomatured-hypoplastic. Another classification is also available which depends upon not only on the 
enamel appearance but also the genetic components which divides the AI into 15 subtypes. These fifteen aforementioned subtypes are the most widely accepted classification so far. Though AI is mainly an enamel related tooth disorder, however, along with this major presentation, other features such as congenital missing teeth, pulp calcifications delayed tooth eruption, root resorption, open bite, negative overjet, and altered vertical jaw relationship were also seen in such patients. ${ }^{4}$ Depending upon the varied clinical presentation, role of different disciplines of dentistry is required for the successful treatment of AI subjects that includes a prosthodontist, a restorative dentist, a maxillofacial surgeon and pediatric dentist. ${ }^{4}$ It is observed that as amelogenesis imperfecta patients presents at younger age hence treatment options usually starts with restorative procedures. So it is recommended to start with minimally invasive procedures i.e direct composite fillings. However literature shows that as the enamel quality of AI patients are compromised therefore direct composite fillings have higher failure rate. ${ }^{5}$ The aim of this case report is to come up with patient friendly restorative treatment for a young female patient with severe AI using indirect composite veneers for anterior and metal crowns on posteriors.

\section{Case Report}

Amelogenesis imperfecta is an enamel formation disorder, diagnosed on the basis of clinical and radiographical findings and gene analysis. A 23 year old adult female patient reported in the OPD of Fatima Memorial hospital on September 2018 with the complaint of sensitivity and poor aesthetics. She was presented with discoloration of the teeth and open bite with poor aesthetics and sensitive teeth. Treatment was started after taking permission from supervisor and Principal of the institute. After the approval a detailed history was recorded about consanguinity and family history of the same problem was recorded to rule out genetic predisposition. Her area of residency was inquired to rule out the high fluoride level. Patient was asked for the gene analysis but she refused because of un affordability, thorough extra oral and intra oral examination revealed the presence of un healthy direct composite fillings on facial surfaces of upper and lower anterior teeth with poor oral hygiene and periodontal condition. As affected subjects of AI are reluctant to smile, speak and socialize themselves because of their overall appearance of face which eventually affects their physiological wellbeing, as they are constantly humiliated by their friends and relatives, so this case highlights the importance of timely and accurate management of amelogenesis imperfecta. The treatment of amelogenesis imperfecta is very costly so the affected patients be should facilitated at Government level which bears high investment on oral aesthetics.

Radiographic findings: Figure no. 1 is showing Orthopantomogram (OPG) on which it was found that tooth \#46 \#47 (Federation Dentire International Classification of teeth) were grossly carious and were not in a condition to be saved anymore as their furcation were involved with minimal coronal tooth structure left, as shown in Fig 2. Hence, she was

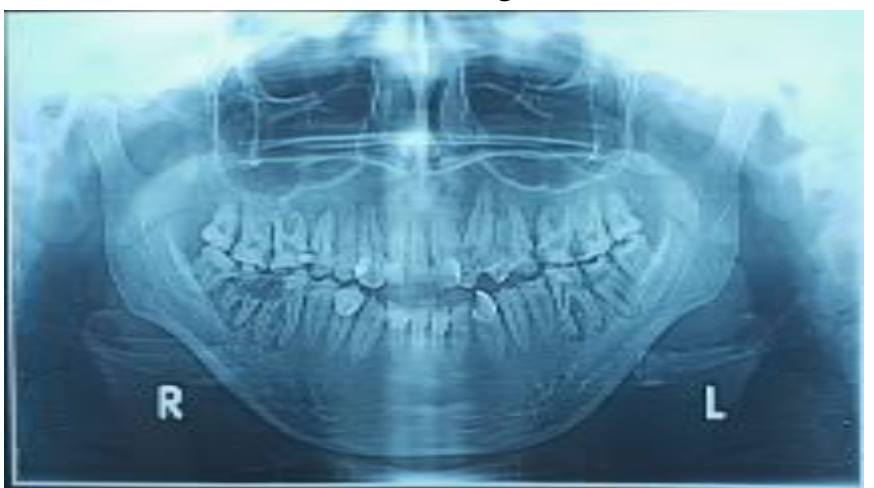

Figure 1: Orthopantomogram

sent to minor oral surgery department for extractions. It was noticed that upper left $\mathrm{c}$ was retained, whereas, upper left canine was impacted in the bone. In Fig 3 the upper right quadrant \#15 \#16 were found carious with pulpal involvement so their root canal treatment (RCT's) were planned. In Fig 4 upper left quadrant \#27 and in Fig 5 \#26 were found grossly carious with pulpal involvement and so their RCT's were planned and executed followed by metal crowns as shown in Fig 10 and Fig 12.
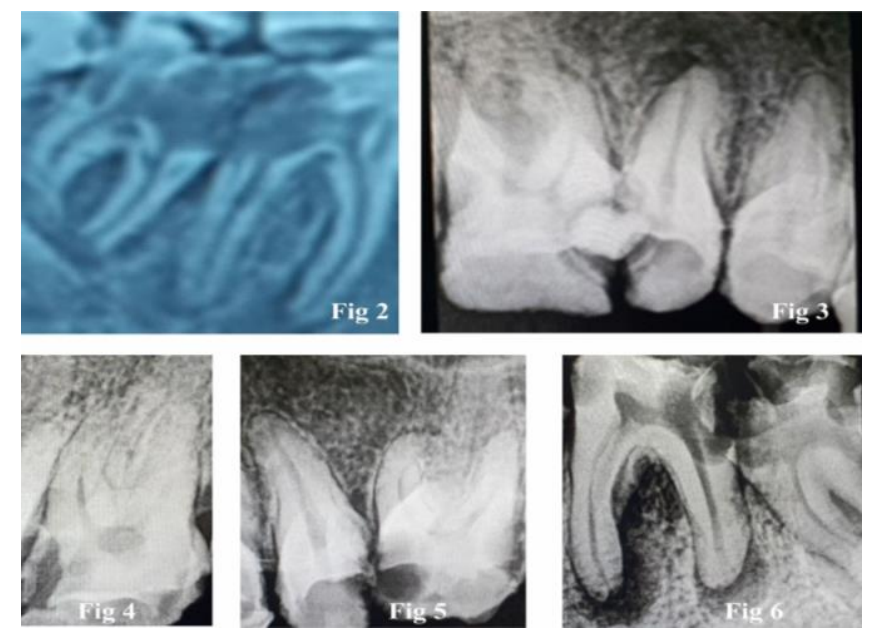

Figures; Periapical pre-treatment X-Rays 


\section{Treatment Adopted}

According to the clinical picture of patient, her ideal treatment plan would have started with Orthodontics to align and correct the bite of upper and lower anterior teeth fully and canine impaction on upper left quadrant prior to any other treatment, but patient had major concerns over a lengthy treatment plan. So our first phase of treatment started with a smile analysis, followed by a full set of photographs, alginate impressions, cast articulation and diagnostic mock up.

Veneer's preparation: Patient's main concern was esthetics. So after smile analysis it was decided to place indirect composite veneers on upper from \#14 to \#24 and lower anteriors from \#34 to \#44 as shown in Fig 7.

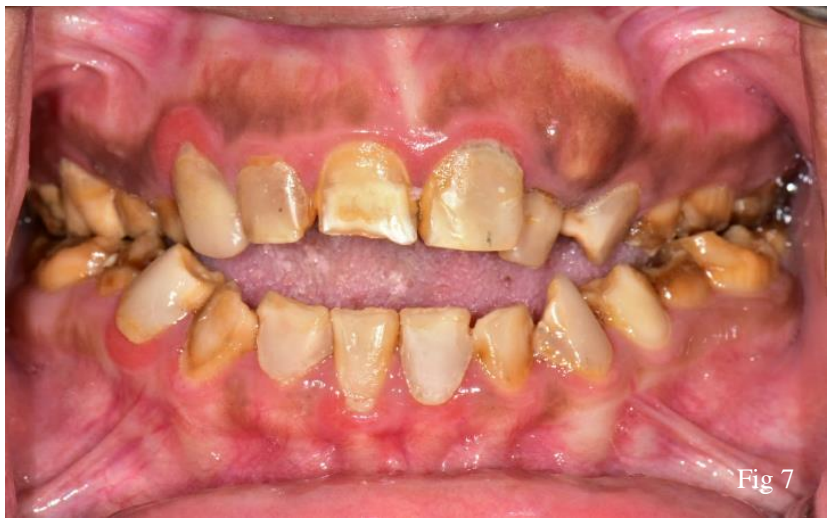

Metal crowns after root canal treatments were given. Veneer preparation was done with tapering fissure bur. First the existing composite restorations were removed then $0.75 \mathrm{~mm}$ cutting

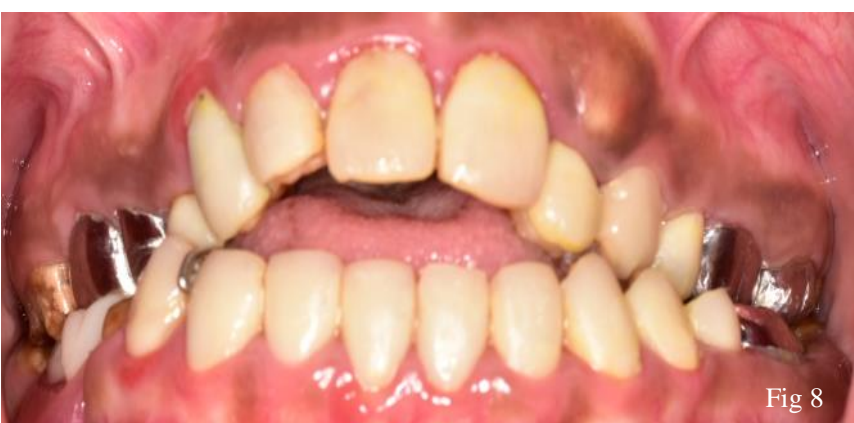

was done. After shade selection putty impression was taken and sent to lab for indirect composite veneers. Veneers were cemented with Relyx cement as shown in Fig 8.
Restorative work: Class 2 amalgam fillings were performed on proximal adjacent sides as can be seen in on Fig 10 and Fig 13 on \#17 and\#25. RCTs were performed on \#15 \#16 \#26 \#27 \#36 and \#37 followed by applying the subject with metal crowns.
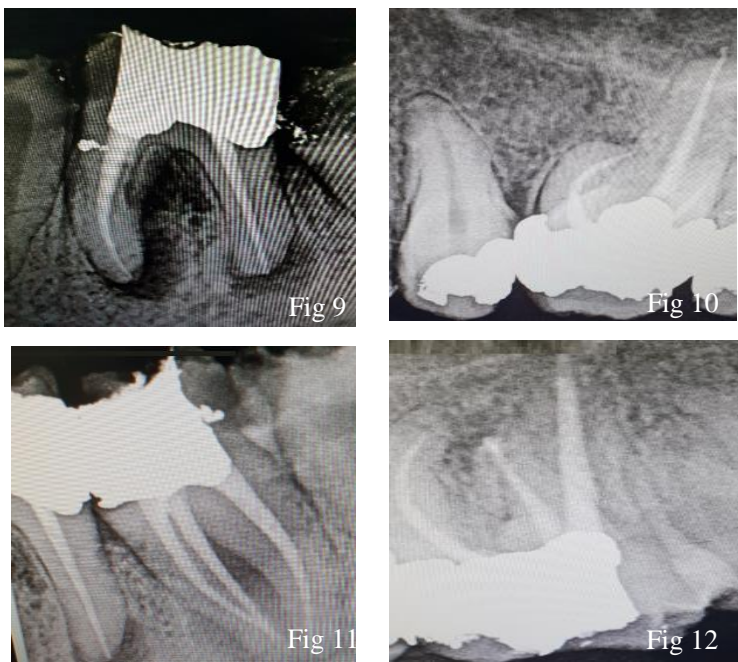

Metal crowns after root canal treatments were given. Patient was referred to prosthodontics department for 2 unit removable partial denture. There her impressions and bite was recorded and sent to the laboratory for manufacturing of partial dentures. After that its trial was done and wax up was sent to lab for final curing. Denture was received after final polishing from the lab and was given to the patient with instructions for its maintenance. It was applied after extractions spaces were healed as shown in Fig 14.
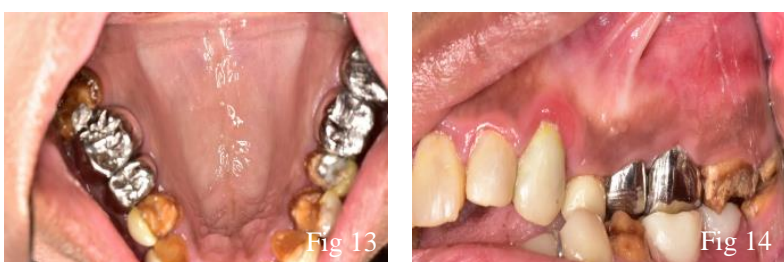

After all the hard work and patients good compliance results made both the patient and her dentist satisfied.

\section{Discussion}

Study done by Parekh et al stated that $77 \%$ of the patients with AI mentioned reported with discoloration and increased sensitivity as a main 
compliant ${ }^{7}$ and this also goes in accordance with the current study presentation. It has been found the main cause of lack of enamel, distorted tooth shape and smaller appearance is exposed dentin, which might be the reason explaining over sensitivity of the dysplastic teeth. ${ }^{8}$ This is also the finding which corresponds to our presentation. Numerious treatment options can be opted for the features and characteristics of AI have been reported. To accomplish acceptable results, multidisciplinary approach is required for the proper execution of treatment plan. In another study $24 \%$ to $64 \%$ of patients affected by AI presented with anterior open bite and mal-occlusions which is in line with our study.

Acknowledgment: I highly acknowledge supervisor Prof. Dr. Saroosh Ehsan and Assistant professor Dr. Shaila Tahir for their unconditional support and guidance.

\section{Conflict of Interest: None}

\section{REFERENCES}

1. Witkop CJ., Jr Amelogenesis imperfecta, dentinogenesis imperfecta and dentin dysplasia revisited: problems in classification. J OralPathol. 1988;17:547553.doi: 10.1111/j.16000714.1988.tb01332.x.)

2. Kim YJ, Kang J, Seymen F, Koruyucu M, Zhang H, Kasimoglu Y, Bayram M, Tuna-Ince EB, Bayrak S, Tuloglu N, Hu JC, Simmer JP, Kim JW. Alteration of Exon Definition Causes Amelogenesis Imperfecta. J Dent Res. 2020 Apr;99(4):410-418. doi: 10.1177/0022034520901708.

3. D Ceyhan, Z Kirzioglu, T Emek .A Long-Term Clinical Study on Individuals with Amelogenesis Imperfecta. Niger J ClinPract.2021;22(8):11571162.doi:10.4103/njcp.njcp2_72_18)

4. Novelli C, Pascadopoli M, Scribante A..Restorative Treatment of Amelogenesis Imperfecta with Prefabricated Composite Veneers. CaseRepDent.2021;2021:3192882.doi:10.1155/2021/3192882.eC ollection 2021
5. B. C. Yaman, F. Ozer, C. S. Cabukusta, M. M. Eren, F. Koray, and M. B. Blatz, "Microtensile bond strength to enamel affected by hypoplastic amelogenesis imperfecta," The Journal of Adhesive Dentistry.2014;16(1);7-14.

6. Sönmez IŞ, Aras Ș, Tunç EŞ, Küçükeșmen Ç. Clinical success of deproteinization in hypocalcified amelogenesis imperfecta. Quintessence International. 2009;40(2).

7. Parekh S, Almehateb M, Cunningham SJ. How do children with amelogenesis imperfecta feel about their teeth? Int J Paediatr Dent. 2014;24:326-335. doi: 10.1111/ipd.12080.

8. Sabandal MMI, Dammaschke T, Schäfer E. Restorative treatment in a case of amelogenesis imperfecta and 9-year follow-up: a case report.Head Face Med. 2020 Nov 19;16(1):28. doi: 10.1186/s13005-020-00243-

9. Koruyucu M, Bayram M, Tuna EB, Gencay K, Seymen F. Clinical findings and long-term managements of patients with amelogenesisimperfecta. EurJDent. 2014;8:546552.doi: 10.4103 1305-7456.143640.

10. Sabandal MM, Dammaschke T, Schäfer E. Restorative treatment in a case of amelogenesis imperfecta and 9-year follow-up: a case report. Head Face Med. 2020; 16: (28). doi: 10.1186/s13005-020-00243-1

\section{Author Contribution}

All authors contributed equally and are responsible for material provided.
Received: 11 Apr 20201,

Revised received: 20 Aug 2021,

Accepted: 30 Sep 2021 